\title{
Translating XML Web Data into Ontologies
}

\author{
Yuan An and John Mylopoulos \\ University of Toronto, Canada \\ \{yuana, jm\}@cs.toronto.edu
}

\begin{abstract}
Translating XML data into ontologies is the problem of finding an instance of an ontology, given an XML document and a specification of the relationship between the XML schema and the ontology. Previous study [8] has investigated the ad hoc approach used in XML data integration. In this paper, we consider to translate an XML web document to an instance of an OWL-DL ontology in the Semantic Web. We use the semantic mapping discovered by our prototype tool [1] for the relationship between the XML schema and the ontology. Particularly, we define the solution of the translation problem and develop an algorithm for computing a canonical solution which enables the ontology to answer queries by using data in the XML document.
\end{abstract}

\section{Introduction}

XML has become an accepted standard for publishing data on the Web. To integrate XML data, a former paper [8] has studied the ad hoc approach to translating various XML documents into a central ontology instance. In this paper, we study a generic and a formal framework for translating an XML document into an instance of an ontology. The following example illustrates the problem. Suppose we have an XML document $\mathcal{X}$ :

$\langle\mathrm{db}>$

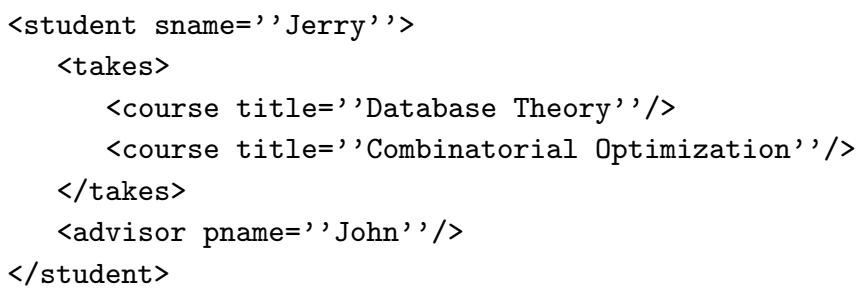

Suppose we have an ontology shown graphically in Figure 1 using UML notation. Given a natural mapping semantically relating the XML schema to the ontology, we would expect that an instance of the ontology contains the following assertions: Student $\left(t_{1}\right)$, Course $\left(t_{2}\right)$, Course $\left(t_{3}\right)$, Professor $\left(t_{4}\right)$, hasName ( $t_{1}$, , Jerry"), hasTitle ( $t_{2}, "$ Data base Theory"), hasTitle ( $t_{3}, "$ Combinatorial Optimization"), hasName $\left(t_{4}, " J o h n "\right)$, takes $\left(t_{1}, t_{2}\right)$, takes $\left(t_{1}, t_{3}\right)$, hasAdvisor $\left(t_{1}, t_{4}\right)$, Professor $\left(u_{1}\right)$, Professor $\left(u_{2}\right)$, Course $\left(u_{3}\right)$, teaches $\left(u_{1}, t_{2}\right)$, teaches $\left(u_{2}, t_{3}\right)$, teaches $\left(t_{4}, u_{3}\right)$, where $t_{i} i=1, \ldots, 4$ and $u_{j} j=1, \ldots 3$ are anonymous individuals in the ontology. 
Note that the difference between $t_{i} \mathrm{~s}$ and $u_{j} \mathrm{~s}$ is that the original XML document provides no information about the individuals $u_{1}, u_{2}$ and $u_{3}$. They were deduced by the ontology constraints. However, if we replace $u_{1}$ and $u_{2}$ by $t_{4}$, then the resulting instance will still satisfy all constraints and it says that professor $t_{4}$ teaches both courses $t_{2}$ and $t_{3}$. Alternatively, we could also construct an instance in which the professor $t_{4}$ teaches only the course $t_{2}$, while the course $t_{3}$ is taught by some unknown professor $u_{2}$. This tells us that there could be different instances that are consistent with the ontology and satisfy a given mapping from the XML schema to the ontology. So if we are given a source document $\mathcal{X}$ shown above and a query over the ontology, how can we answer it? If our query is, for example, What is the name of the person who is the advisor of the person whose name is Jerry? The answer is John regardless of a particular instance that was created for the ontology. As another example, consider the query What is the title of the course taught by Jerry's advisor? This query cannot be answered with certainty in this scenario.

Ontologies play a central role in the Semantic web. Recently, W3C has recommended the OWL web ontology language for describing ontologies in the Semantic Web. If an XML document needs to

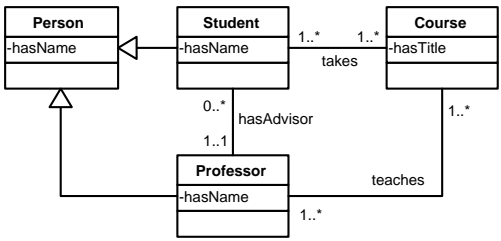

Fig. 1. An Ontology ontology, the resulting ontology should preserve the information in the XML document and be able to answer queries by using these information. Consequently, a translation involves specifying a mapping, checking the consistency, and preserving information. In this paper, we consider the OWL-DL ontology language because of its close relationship with Description Logics. As a result, the OWL-DL ontology language enable us to develop a translation algorithm. The overall framework is generic in the sense that the theoretical issues apply to many translation problems between databases and ontologies.

The rest of the paper is organized as follows. Section 2 presents the formal specifications about OWL-DL ontology and XML. Section 3 defines the problem and Section 4 defines the canonical solution. Section 5 develops the algorithm, and finally, Section 6 gives the conclusions.

\section{Preliminaries}

We assume readers are familiar with the standard notations and semantics of Description Logics, though we summarize here one flavor relating to the OWL-DL web ontology language. OWL-DL is closely related to the $\mathcal{S H O I N}(\mathbf{D})$ description logic [5], and the meanings of its terminology can be found in $[4,5]$.

A datatype theory $\mathbf{D}$ is a mapping from a set of datatypes to a set of values. The datatype (or concrete) domain, written $\Delta_{\mathbf{D}}^{\mathcal{I}}$, is the union of the mappings 
of the datatypes. Let $\mathbf{R}$ be set of role names consisting of a set of abstract role names $\mathbf{R}_{A}$ and a set of concrete role names $\mathbf{R}_{D}$. The set of $\mathcal{S H O I N}$-roles (or roles for short) consist of a set of abstract roles $\mathbf{R}_{A} \cup\left\{R^{-} \mid R \in \mathbf{R}_{A}\right\}$ and a set of concrete roles $\mathbf{R}_{D}$. An $R$ Box $\mathcal{R}$ consists of a finite set of transitivity axioms $\operatorname{Trans}(R)$, and role inclusion axioms of the form $R \sqsubseteq S$ and $T \sqsubseteq U$, where $R$ and $S$ are abstract roles, and $T$ and $U$ are concrete roles. $\complement^{*}$ denotes the reflexivetransitive closure of $\sqsubseteq$ on roles, i.e., for two abstract roles $R, S, S \sqsubseteq^{*} R \in \mathcal{R}$ if $S$ and $R$ are the same, $S \sqsubseteq R \in \mathcal{R}, \operatorname{lnv}(S) \sqsubseteq \operatorname{lnv}(R) \in \mathcal{R}$, or there exists some role $Q$ such that $S \sqsubseteq^{*} Q \in \mathcal{R}$ and $Q \sqsubseteq^{*} R \in \mathcal{R}$. A role not having transitive sub-roles is called a simple role, and $\operatorname{Inv}(R)=R^{-}$.

The set of $\mathcal{S H O I N}(\mathbf{D})$ concepts is defined by the following syntactic rules, where $C_{i}$ s are concepts, $A$ is an atomic concept, $R$ is an abstract role, $S$ is an abstract simple role, $T$ is a concrete role, $o_{i}$ are individuals, $D$ is a datatype, and $n$ is a non-negative integer:

$$
\begin{aligned}
C \rightarrow & A|\neg C| C_{1} \sqcap C_{2}\left|C_{1} \sqcup C_{2}\right| \exists R . C|\forall R . C| \geq n S|\leq n S| \\
& \left\{o_{1}, . ., o_{n}\right\}|\geq n T| \leq n T|\exists T . D| \forall T . D
\end{aligned}
$$

A $T B o x \mathcal{T}$ consists of a finite set of concept inclusion axioms $C_{1} \sqsubseteq C_{2}$; an $A B o x \mathcal{A}$ consists of a finite set of concept and role assertions and individual (in)equalities $C(a), R(a, b), a=b, a \neq b$, respectively. A $\mathcal{S H O I N}(\mathbf{D})$ knowledge base (an ontology) $\mathcal{O}=(\mathcal{T}, \mathcal{R}, \mathcal{A})$ consists of a TBox $\mathcal{T}$, an RBox $\mathcal{R}$, and

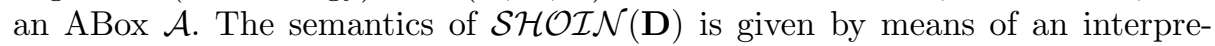
tation $\mathcal{I}=\left(\Delta^{\mathcal{I}},{ }^{\mathcal{I}}\right)$ consisting of an non-empty domain $\Delta^{\mathcal{I}}$, disjoint from the datatype domain $\Delta_{\mathbf{D}}^{\mathcal{I}}$, and a mapping ${ }^{\mathcal{I}}$, which interprets atomic and complex concepts, roles, axioms, and assertions in the standard description logic way. An interpretation $\mathcal{I}$ is a model of the knowledge base $\mathcal{O}=(\mathcal{T}, \mathcal{R}, \mathcal{A})$ if $\mathcal{I}$ satisfies every concept, axiom, and assertion in $\mathcal{O}$. From the database perspective, the TBox $\mathcal{T}$ and the RBox $\mathcal{R}$ can be viewed as a schema with unary and binary relational tables, and the $\mathrm{ABox} \mathcal{A}$ can be viewed as an instance. An $\mathrm{ABox} \mathcal{A}$ is consistent with respect to $\mathcal{O}$ if there is a model of $(\mathcal{T}, \mathcal{R}, \mathcal{A}$ ) (we say $\mathcal{O}$ is consistent). A concept or role assertion $\beta$ is a logical consequence of an ABox $\mathcal{A}$ (written $\mathcal{A}=\beta$ ), if for every model of $\mathcal{A}$ w.r.t $\langle\mathcal{T}, \mathcal{R}\rangle, \beta$ is true. We write $\mathcal{O} \models \beta$ for $\beta$ is a logical consequence of the ontology.

An XML document is typically modeled as a node-labeled tree. For our purpose, we assume that each XML document is described by an XML schema consisting of a set of element and attribute type definitions. Specifically, we assume the following countably infinite disjoint sets: Ele of element names, Att of attribute names, and Dom of simple type names including the built-in XML schema datatypes. Attribute names are preceded by a "@" to distinguish them from element names. Given finite sets $E \subset$ Ele and $A \subset$ Att, a XML schema $\mathcal{S}=(E, A, \tau, \rho, \kappa)$ specifies the type of each element $\ell$ in $E$, the attributes that $\ell$ has, and the datatype of each attribute in $A$. Specifically, An element type $\tau$ is defined by the grammar $\tau::=\epsilon \mid$ Sequence $\left[\ell_{1}: \tau_{1}, \ldots \ell_{n}: \tau_{n}\right] \mid$ Choice $\left[\ell_{1}: \tau_{1}, . ., \ell_{n}: \tau_{n}\right]$ $\left(\ell_{1}, . ., \ell_{n} \in E\right)$, where $\epsilon$ is for the empty type, and Sequence and Choice are complex types. Each element associates an occurrence constraint with two values: 
minOccurs indicating the minimum occurrence and maxOccurs indicating the maximum occurrence. The set of attributes of an element $\ell \in E$ is defined by the function $\rho: E \rightarrow 2^{A}$; and the function $\kappa: A \rightarrow$ Dom specifies the datatypes of attributes in $A$. Each datatype name associates with a set of values in a domain Dom. In this paper, we do not consider the simple type elements (corresponding to DTD's PCDATA ), assuming instead that they have been represented using attributes. Furthermore, a special element $\underline{r} \in E$ is the root of the XML schema such that $\rho(\underline{r})=\emptyset$, and we assume that for any two element $\ell_{i}, \ell_{j} \in E$, $\rho\left(\ell_{i}\right) \cap \rho\left(\ell_{j}\right)=\emptyset$.

An XML document $\mathcal{X}=(N,<, \underline{r}, \lambda, \eta)$ over $(E, A)$ consists of a set of nodes $N$, a child relation $<$ between nodes, a root node $\underline{r}$, and two functions such as:

- a labeling function $\lambda: N \rightarrow E \cup A$ such that if $\lambda(v)=\ell \in E$, we say that $v$ is in the element type $\ell$; if $\lambda(v)=@ a \in A$, we say that $v$ is an attribute @ $a$;

- a partial function $\eta: N \rightarrow D o m$ for every node $v$ with $\lambda(v)=@ a \in A$, assigning values in domain Dom that supplies values to simple type names in Dom.

An XML document $\mathcal{X}=(N,<, \underline{r}, \lambda, \eta)$ conforms to a schema $\mathcal{S}=(E, A, \tau, \rho, \kappa)$, denoted by $\mathcal{X} \models \mathcal{S}$, if:

1. for every node $v$ in $\mathcal{X}$ with children $v_{1}, . ., v_{m}$ such that $\lambda\left(v_{i}\right) \in E$ for $i=$ $1, \ldots, m$, if $\lambda(v)=\ell$, then $\lambda\left(v_{1}\right), \ldots, \lambda\left(v_{m}\right)$ satisfies $\tau(\ell)$ and the occurrence constraints.

2. for ever node $v$ in $\mathcal{X}$ with children $u_{1}, \ldots, u_{n}$ such that $\lambda\left(u_{i}\right)=@ a_{i} \in A$ for $i=1, \ldots, n$, if $\lambda(v)=\ell$, then $\lambda\left(u_{i}\right)=@ a_{i} \in \rho(\ell)$, and $\eta\left(u_{i}\right)$ is a value having datatype $\kappa\left(@ a_{i}\right)$.

Now we turn to the mapping language relating a pattern in an XML schema with a formula in an ontology. On the XML side, the basic component is attribute formulas [2], which are specified by the syntax $\alpha::=\ell \mid \ell\left(@ a_{1}=x_{1}, . ., @ a_{n}=x_{n}\right)$, where $\ell \in E$, @ $a_{1}, . ., @ a_{n} \in A, E$ and $A$ are element names and attribute names respectively; and variables $x_{1}, . ., x_{n}$ are the free variables of $\alpha$. Treepattern formulas over an XML schema $\mathcal{S}=(E, A, \tau, \rho, \kappa)$ are defined by $\psi::=$ $\alpha \mid \alpha\left[\varphi_{1}, . ., \varphi_{n}\right]$, where $\alpha$ ranges over attribute formulas over $(E, A)$. The free variables of a tree formula $\psi$ are the free variables in all the attribute formulas that occur in it. For example, Company[Department [employee(@eid $\left.=x_{1}\right)[$ manager $\left(@\right.$ mid $\left.=x_{2}\right)$ [employee $\left.\left.\left.\left(@ e i d=x_{3}\right)\right]\right]\right]$ is a tree formula.

An attribute formula is evaluated in a node of an XML document, and values for free variables come from domain $D o m$. If $\mathcal{X}$ is an XML document over $(E, A)$ and $v$ a node of $\mathcal{X}$, then

$-(\mathcal{X}, v) \models \ell$ iff $\lambda(v)=\ell$, for $\ell \in E$.

- if $\alpha\left(x_{1}, \ldots, x_{n}\right)=\ell\left(@ a_{1}=x_{1}, . ., @ a_{n}=x_{n}\right)$, then $(\mathcal{X}, v) \models \alpha\left(s_{1}, \ldots, s_{n}\right)$, where $s_{1}, \ldots, s_{n} \in D o m$, iff $\lambda(v)=\ell$, and for each child $v_{i}$ of $v$ such that $\lambda\left(v_{i}\right)=@ a_{i}$, $\eta\left(v_{i}\right)=s_{i}$ for $i \in[1, . . n]$. 
Given a document $\mathcal{X}$, a tree-pattern formula $\psi(\bar{x})$, and a tuple $\bar{s}$ from Dom, $\psi(\bar{s})$ is satisfied in $\mathcal{X}$ (written $\mathcal{X} \models \psi(\bar{s})$ ) if there is a witness node $v$ for $\psi(\bar{s})$. Formally, a witness node for a $\psi(\bar{s})$ is defined as follows:

$-v$ is a witness node for $\alpha(\bar{s})$, where $\alpha$ is an attribute formula, iff $(\mathcal{X}, v) \models \alpha(\bar{s})$.

$-v$ is witness node for $\alpha(\bar{s})\left[\psi_{1}\left(\bar{s}_{1}\right), \ldots, \psi_{m}\left(\bar{s}_{m}\right)\right]$ iff $(\mathcal{X}, v) \models \alpha(\bar{s})$ and there are $m$ children $v_{1}, \ldots, v_{m}$ of $v$ such that each $v_{i}$ is a witness node for $\psi_{i}\left(\bar{s}_{i}\right)$, for $i=1, \ldots, m$.

On the ontology side, we use conjunctive formulas with annotations, which treat atomic concepts and roles as unary and binary predicates, respectively. For example, given an ontology containing the atomic concept Employee and roles hasId, hasManager, and manages, the following is a mapping formula, Company[Department [

$$
\begin{aligned}
& \text { employee }\left(@ e i d=x_{1}\right)[ \\
& \text { manager }\left(@ \text { mid }=x_{2}\right)[
\end{aligned}
$$$$
\text { employee (@eid=x } x_{3} \text { ) ]]]] } \rightarrow
$$

Employee $\left(Y_{1}\right), \operatorname{hasId}\left(Y_{1}, x_{1}\right)$, Employee $\left(Y_{2}\right), \operatorname{hasId}\left(Y_{2}, x_{2}\right)$,

hasManager $\left(Y_{1}, x_{2}\right)$, Employee $\left(Y_{3}\right), \operatorname{hasId}\left(Y_{3}, x_{3}\right)$, manages $\left(Y_{2}, Y_{3}\right):$ :

identif $\left(Y_{1}, x_{1}\right)$, identif $\left(Y_{2}, x_{2}\right)$, identif $\left(Y_{3}, x_{3}\right)$.

There are two sorts of variables. One sort of variables denoted, e.g., by $Y_{i} \mathrm{~s}$, represent the individuals in the ontology, and another sort of variables denoted, e.g., by $x_{j}$ s, represent data values containing the attribute values in the XML document and concrete values in the ontology. Since attribute values in the XML document come from the domain Dom, while concrete values in the ontology come from domain $\Delta_{\mathbf{D}}^{\mathcal{I}}$, we assume that each mapping formula implies a set of conversion functions such that when the single variable name $x_{j}$ is used on both sides, both datatypes in the corresponding positions are matched through an implicit conversion function. We denote by ConstValue the set of all data values that occur in the XML document and we also call them constant values. In addition, we assume an infinite set VarValue which we call variable values including an infinite set Individual of individuals and an infinite set DataValue of data values. We require that ConstValue $\underline{\text { VarValue }}=\emptyset$.

The annotation comes after :: in the mapping formula. Each predicate in the annotation is of the form $\operatorname{identif}(Y, \bar{Z})$ in which $Y$ is an individual variable and $\bar{Z}$ is a tuple of variables. The meaning of identif $(Y, \bar{Z})$ is as follows. The information in XML document indicates that an individual belonging to the concept $C$ in which $Y$ is the placeholder variable, i.e, $C(Y)$ appearing in the formula, can be identified by a set of roles $P_{1}, \ldots, P_{n}$ in the ontology, whereas $P_{1}, \ldots, P_{n}$ bind $Y$ with $\bar{Z}$ in the formula, i.e., $P_{1}\left(Y, Z_{1}\right), \ldots, P_{n}\left(Y, Z_{n}\right)$ appear. We will see later that the annotation is important during the translation and for consistency checking in the ontology. To specify the mapping formulas, we have proposed a semi-automatic tool MAPONTO in [1].

\section{The Problem of Translating XML data into Ontologies}

We now define the problem of translating XML into ontologies (X-to-O). 
Definition 1 (Semantics of Mapping Formulas). Given an XML schema $\mathcal{S}$ and an ontology $\mathcal{O}=<\mathcal{T}, \mathcal{R}, \mathcal{A}>$, a mapping formula is an expression of the form:

$$
\Psi: \psi(\bar{x}) \rightarrow \varphi(\bar{Y}, \bar{x}):: \text { annotation. }
$$

where $\psi(\bar{x})$ is a tree-pattern formula over $\mathcal{S}, \varphi(\bar{Y}, \bar{x})$ is a conjunctive formula over atomic concept and role names of $\mathcal{O}$, and $\bar{Y}$ and $\bar{x}$ have no variables in common.

Given an $X M L$ document $\mathcal{X}$ conforming to $\mathcal{S}$ and an ontology instance $\mathcal{A}$ consistent with $\mathcal{O}$, we say that the pair $\langle\mathcal{X}, \mathcal{A}\rangle$ satisfies the formula (1) if whenever there is a tuple $\bar{s}$ such that $\mathcal{X} \models \psi(\bar{s})$, there exists a tuple $\bar{t}$ such that for each assertion $\beta$ in the formula $\varphi(\bar{t}, \bar{s}), \mathcal{A} \models \beta$.

Definition 2 (X-to-O problem). The problem of translating XML data into ontologies (X-to-O) is a triple $\left(\mathcal{S}, \mathcal{O}, \Sigma_{\mathcal{S O}}\right)$, where $\left.\mathcal{S}=<E, A, \tau, \rho, \kappa\right\rangle$ is an $X M L$ schema, $\mathcal{O}=<\mathcal{T}, \mathcal{R}, \mathcal{A}>$ is an ontology, and $\Sigma_{\mathcal{S O}}$ is a set of mapping formulas between $\mathcal{S}$ and $\mathcal{O}$.

Definition 3 (Solutions). Given an $X$-to-O problem $\mathcal{P}=\left(\mathcal{S}, \mathcal{O}, \Sigma_{\mathcal{S O}}\right)$ and an $X M L$ document $\mathcal{X}$ conforming to $\mathcal{S}$, an instance $\mathcal{A}$ consistent with $\mathcal{O}$ such that $<\mathcal{X}, \mathcal{A}\rangle$ satisfies all formulas in $\Sigma_{\mathcal{S O}}$ is called a solution for $\mathcal{P}$.

Recall the Data Exchange problem [3,2]. A data exchange setting is a tuple $\left(\mathbf{S}, \mathbf{T}, \Sigma_{s t}, \Sigma_{t}\right)$, where $\mathbf{S}$ is a source schema, $\mathbf{T}$ is a target schema, $\Sigma_{s t}$ is a set of source-to-target dependencies, or STDs, that express the relationship between $\mathbf{S}$ and $\mathbf{T}$, and $\Sigma_{t}$ is a set of constraints on the target schema. A solution of the data exchange problem is an instance $J$ over the target schema $\mathbf{T}$ when given an instance $I$ over the source schema $\mathbf{S}$, such that $I$ and $J$ together satisfy all formulas in $\Sigma_{s t}$ and $\Sigma_{t}$. In general, there may be many different solutions for a given instance $I$, and under target constraints, there may be no solutions at all. If one poses query $Q$ over the target schema, and a source instance $I$ is known, the usual semantics in data exchange uses certain answers. A key problem in data exchange is to find a particular solution $J_{0}$ so that $\underline{\text { certain }}(Q, I)$ can be obtained by evaluating some query over $J_{0}$.

Coming back to X-to-O problem, we have defined that the source is an XML schema and the target is an ontology. By analogy, our mapping formulas are the source-to-target dependencies that express the relationships between the XML schema and the ontology. Given an XML document conforming to the XML schema, we want to compute a consistent instance of the ontology, such that the XML document together with the ontology instance satisfy all formulas in the mapping. The major difference from the data exchange problem is that with an ontology as the target, computing a solution calls for a different algorithm.

\section{Canonical Solution}

As illustrated by the example in Section 1, there could be many solutions for an X-to-O problem. In this section, we define the canonical solution in terms of 
answering queries against ontologies. For the query language, we use a simple conjunctive query language $\left(\mathrm{CQ}_{0}\right)$ which can represent most of the proposed query languages for RDF data (e.g., SPARQL [7]). Formally, a CQo query is of the form:

$$
Q: q(\bar{x}) \leftarrow p_{1}\left(\overline{Y_{1}}\right), p_{2}\left(\overline{Y_{2}}\right), \ldots, p_{n}\left(\overline{Y_{n}}\right) .
$$

where $\bar{x}$ is a tuple of variables or constants which take values from concrete (datatype) domains (e.g., Integer, String, etc.), $\overline{Y_{1}}, \ldots, \overline{Y_{n}}$ are tuples of variables or constants which take values from both concrete domains and individuals (e.g., object identifiers), and we require $\bar{x} \subset \overline{Y_{1}} \cup \ldots \cup \overline{Y_{n}}$. The predicates $p_{1}, \ldots, p_{n}$ are atomic concept and (abstract and concrete) role names in an ontology. The predicate $q$ is an ordinary predicate with an arity $m=|\bar{x}|$. Let $\underline{\text { Const denote }}$ the set of constants appearing in an ontology, Var denote a set of variables, and $\underline{\text { Const }} \cap \underline{\operatorname{Var}}=\emptyset$. Let $h: \underline{\text { Const }} \cup \underline{\mathrm{Var}} \rightarrow \underline{\text { Const }}$ be a mapping from a tuple of variables or constants to a tuple of constants such that if $c \in \underline{\text { Const }}, h(c)=c$. Given an ontology $\mathcal{O}=\langle\mathcal{T}, \mathcal{R}, \mathcal{A}>$ with an instance $\mathcal{A}$, the answer of the query (2) is defined as a set of tuples of concrete (datatype) values $\{\bar{s}\}$ such that for each tuple $\bar{s}$ there is a mapping $h$ such that $h(\bar{x})=\bar{s}$ and there are tuples $h\left(\overline{Y_{i}}\right)$, $\mathcal{O} \models p_{i}\left(h\left(\overline{Y_{i}}\right)\right)$ for each $i=1, \ldots, n$. A $\mathrm{CQ}_{0}$ query only returns tuples consisting of datatype values.

Assume that we are given an X-to-O problem $\left(\mathcal{S}, \mathcal{O}, \Sigma_{\mathcal{S O}}\right)$, an XML document $\mathcal{X}$ conforming to $\mathcal{S}$, and a $\mathrm{CQ}_{0}$ query $Q$ against the ontology. What does it mean to answer $Q$ ? As in the data exchange problem [3], since there may be many possible solutions to the X-to-O problem, we define the semantics of $Q$ in terms of certain answers:

$$
\underline{\operatorname{certain}}(Q, \mathcal{X})=\bigcap_{\mathcal{A}^{\prime} \text { is a solution }} Q\left(\mathcal{A}^{\prime}\right)
$$

where, $Q\left(\mathcal{A}^{\prime}\right)$ is the answers of the query $Q$ evaluated over the solution $\mathcal{A}^{\prime}$. Thus, a tuple $\bar{s}$ of datatype values is in the set of certain answers certain $(Q, \mathcal{X})$, if $\bar{s} \in Q\left(\mathcal{A}^{\prime}\right)$ for every solution $\mathcal{A}^{\prime}$ of the X-to-O problem.

Definition 4 (Canonical Solution). Given an X-to-O problem and a $C Q_{0}$ query $Q$ against the ontology. A solution $\mathcal{A}$ is a canonical solution if it produces the certain answers when given an XML document $\mathcal{X}$ conforming to the XML schema.

\section{Computing a Canonical Solution}

Given an X-to-O problem $\mathcal{P}=\left(\mathcal{S}, \mathcal{O}, \Sigma_{\mathcal{S O}}\right)$, we assume that the ontology $\mathcal{O}=<$ $\mathcal{T}, \mathcal{R}, \mathcal{A}>$ is satisfiable. For each mapping formula $\Psi: \psi(\bar{x}) \rightarrow \varphi(\bar{Y}, \bar{x})::$ annotation, the formula $\varphi(\bar{Y}, \bar{x})$ has the form $C_{i}\left(Y_{i}\right), \ldots, P_{i}\left(Y_{i}, Y_{j}\right), \ldots, T_{i}\left(Y_{i}, x_{i_{j}}\right), \ldots$ where $C_{i}$ is an atomic concept name, $P_{i}$ is an abstract role name, $T_{i}$ is a concrete role name, and $\bar{x}=\left\{\ldots x_{i_{j}} \ldots\right\}$. We assume that $\varphi(\bar{Y}, \bar{x})$ is consistent with the ontology $\mathcal{O}$ (written $\mathcal{O} \models \varphi(\bar{Y}, \bar{x})$ ), which means that for each model $\mathcal{I}=<\Delta^{\mathcal{I}},{ }^{\mathcal{I}}>$ 
of $\mathcal{O}$, the interpretation function ${ }^{\mathcal{I}}$ can be extended to the variables in $\varphi(\bar{Y}, \bar{x})$ in such a way that $\mathcal{I}$ satisfies every atom in $\varphi\left(\bar{Y}^{\mathcal{I}}, \bar{x}^{\mathcal{I}}\right)$.

Informally, to compute a canonical solution when given a mapping formula $\Psi$ and an XML document $\mathcal{X}$, we start from an initial ontology $\mathcal{O}_{0}=<\mathcal{T}, \mathcal{R}$, $\mathcal{A}_{0}>$ and add new assertions $C_{i}\left(t_{i}\right), P_{i}\left(t_{i}, t_{j}\right)$, and $T_{i}\left(t_{i}, s_{i_{j}}\right)$ in turn to generate a series of ABoxes $\mathcal{A}_{1}, \mathcal{A}_{2}, \mathcal{A}_{3} \ldots$, whenever there is a tuple $\bar{s} \in$ ConstValue such that $\mathcal{X} \models \psi(\bar{s})$. The assertions $C_{i}\left(t_{i}\right), P_{i}\left(t_{i}, t_{j}\right)$, and $T_{i}\left(t_{i}, s_{i_{j}}\right)$ are instantiated from the mapping formula by substituting $\bar{s}$ for $\bar{x}$ and by substituting $\bar{t}$ for $\bar{Y}$, where $\bar{t}$ is a tuple of values in Individual. When adding these assertions, some extra assertions will probably be added according to axioms in TBox and RBox. There will be a finite number of ABoxes $\mathcal{A}_{1}, \mathcal{A}_{2}, \ldots, \mathcal{A}_{n}$ because there are finite number of tuples in $\mathcal{X}$ satisfying the mapping formula and the propagation we will use terminates. Before presenting the algorithm, we first describe how to generate $\bar{t}$.

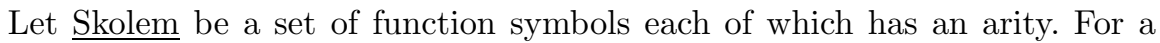
function symbol $f_{C}$ of arity $n$ w.r.t. the concept $C$, the value of applying $f_{C}$ to a set of values $d_{1}, \ldots, d_{n} \in \underline{\text { ConstValue}} \cup$ Individual is denoted as $f_{C}\left(d_{1}, . ., d_{n}\right)$. We require that $f_{C}\left(d_{1}, . ., d_{n}\right)$ is in the set Individual and two $f_{C}\left(d_{1}, \ldots, d_{n}\right)$ are equal iff they are syntactically equivalent. We choose $\bar{t}$ as follows. Suppose the annotation of the formula $\Psi$ has a predicate $i d e n t i f\left(Y_{i}, \bar{Z}\right)$, where $C_{i}\left(Y_{i}\right)$ is in $\Psi$. If $\bar{Z}$ consists of only variables in $\bar{x}$, then let $t_{i}=f_{C_{i}}([\bar{Z} / \bar{s}])([\bar{Z} / \bar{s}]$ means substituting $\bar{s}$ for the variables in $\bar{Z}$ w.r.t. the substitution of $\bar{x}$ in $\varphi(\bar{Y}, \bar{x}))$; else $t_{i}=f_{C_{i}}\left(\left[\bar{Z} / \bar{s} \cup \overline{t_{j}}\right]\right)$, where each $t_{j}$ is an individual chosen recursively for individual variables in $\bar{Z}$. The process terminates due to the propagation of the tree structures in XML documents in the annotation.

To detect any inconsistent ABox during the process of computing the canonical solution, we add extra assertions in addition to the assertions instantiated from the mapping formula. A set of propagation rules serves this purpose. The propagation rules are derived from the axioms in the TBox and RBox, and they only apply to the individuals constructed by Skolem functions. We assume that all inclusion axioms in TBox are concept definition and the TBox is acyclic. That means only axioms of the form $C N \sqsubseteq C$ or $C N \doteq C$ are in TBox, where $C N$ is a concept name, and $C$ does not directly or indirectly refer to $C N$.

Here are the propagation rules. For an ABox $\mathcal{A}_{i}$ and individuals $t$ of the form $f_{C}(\bar{s})$ where $f_{C}$ is a Skolem function symbol, we add new assertions which do not exist previously to $\mathcal{A}_{i}$ by the following rules:

1. adding $C(t)$ if $C N(t)$ and $C N \sqsubseteq C$ or $C N \doteq C$ are in TBox;

2. adding $C_{1}(t)$ and $C_{2}(t)$ if $\left(C_{1} \sqcap C_{2}\right)(t)$ is in $\mathcal{A}_{i}$;

3. adding $C\left(t_{1}\right)(v \in d)$ if $(\forall R . C)(t)$ and $R\left(t, t_{1}\right)$ (resp. $(\forall T . d)(t)$ and $\left.T(t, v)\right)$ are in $\mathcal{A}_{i}$;

4. adding $R(t, u)$ and $C(u)(T(t, v)$ and $v \in d)$ if $(\exists R . C)(t)$ (resp. $(\exists T . d)(t))$ is in $\mathcal{A}_{i}$ and there is no $R(t, u)$ (resp. $T(t, v)$ );

5. adding $P\left(t, t_{1}\right)$ (or $P\left(t_{1}, t\right)$ ) if $R\left(t, t_{1}\right)$ (or $R\left(t_{1}, t\right)$ ) is in $\mathcal{A}_{i}$ and $R \sqsubseteq^{*} P$ in RBox;

6. replacing $t$ with one of $\left\{o_{1}, \ldots, o_{n}\right\}$ if $\left\{o_{1}, \ldots, o_{n}\right\}(t)$ is in $\mathcal{A}_{i}$; 
7. adding $C_{1}(t)$ or $C_{2}(t)$ if $\left(C_{1} \sqcup C_{2}\right)(t)$ is in $\mathcal{A}_{i}$;

8. replacing the occurrences of $t_{i}$ with $t_{j}$ for an individual $t_{i}$ not computed by a Skolem function and an individual $t_{j}$ of the form $f_{C}(\bar{s})$, if $(\leq n R)(t)$ is in $\mathcal{A}_{i}$ and there exists $t_{k} k=1, \ldots, n+1$ such that $R\left(t, t_{k}\right)$ exists.

Rules 1-5 above are deterministic and rules 6-8 are nondeterministic. There is only one generating rule 4.) which generates $u$ from VarValue but $u$ does not use Skolem functions; therefore, the propagation will terminate for a tree structure with depth at most 1 .

A number restriction clash is the situation in that some abstract role $R$ (resp. concrete role $T),(\leq n R)(t)$ (resp. $(\leq n T)(t))$ is in $\mathcal{A}_{i}$ and there are $n+1$ different individuals $t_{1}, \ldots, t_{n+1}\left(\right.$ resp. values $\left.v_{1}, \ldots, v_{n+1}\right)$ such that $R\left(t, t_{j}\right)$ (resp. $T\left(t, v_{j}\right)$ ) in $\mathcal{A}_{i}$ for $j=1, \ldots, n+1$. If an $\mathrm{ABox} \mathcal{A}_{i}$ contains a number restriction clash when adding assertions either by instantiating the mapping formula or by applying the propagation rules, then no canonical solution exists and the algorithm returns immediately with an empty solution. Otherwise, the algorithm terminates and generates a series of consistent ABoxes $\mathcal{A}_{0}, \mathcal{A}_{1}, \ldots, \mathcal{A}_{n}$. Starting from a consistent initial $\mathrm{ABox} \mathcal{A}_{0}$, for the last $\mathrm{ABox} \mathcal{A}_{n}$, we have the following property:

Lemma 1. $\mathcal{A}_{n}$ is a solution.

Proof. (Sketch) $\mathcal{A}_{n}$ contains all assertions which can be constructed from the mapping formulas and tuples $\bar{s}$ such that $\mathcal{X} \models \psi(\bar{s})$. What we need to prove is that $\mathcal{A}_{n}$ is consistent. To do this, we will use the sound and complete tableaux algorithm for deciding $\mathcal{S H O I N}(\mathbf{D})$ knowledge bases. The algorithm is shown in the papers $[4,5]$. They show that if a knowledge base is satisfiable, then the algorithm does not generate any clashes.

The propagation rules used in our algorithm for computing a canonical solution is a subset of the expansion rules for deciding $\mathcal{S H O I N}(\mathbf{D})$ knowledge bases. However, in contrast to application of the expansion rules, our propagation rules only apply to individuals computed from the mapping formula and tuples in the XML document. Each individual has the form of $f_{C}(\bar{s})$.

Then it suffices to prove that if $\mathcal{A}_{n}$ does not contains the number restriction clash, then the decision procedure does not generate any clashes.

Further, we have

Proposition 1. $\mathcal{A}_{n}$ is a canonical solution. That is, given an $X M L$ document $\mathcal{X}$ and a $C Q_{0}$ query $Q, Q\left(\mathcal{A}_{n}\right)=\underline{\operatorname{certain}}(Q, \mathcal{X})$.

Proof. Suppose $Q$ is $q(\bar{x}) \leftarrow p_{1}\left(\overline{Y_{1}}\right), \ldots, p_{n}\left(\overline{Y_{n}}\right)$.

Lemma 1 has shown that $\mathcal{A}_{n}$ is a solution.

Let $\bar{s}$ be a tuple of data values. If $\bar{s} \in Q\left(\mathcal{A}_{n}\right)$, then there is a mapping $h$ from $\bar{x}$ to $\bar{s}$ such that $\left(\mathcal{T}, \mathcal{R}, \mathcal{A}_{n}\right) \models p_{i}\left(h\left(\overline{Y_{i}}\right)\right)$ for each $i$. We need to prove that for every solution $\mathcal{A}, \bar{s} \in Q(\mathcal{A})$, i.e., $(\mathcal{T}, \mathcal{R}, \mathcal{A}) \models p_{i}\left(h\left(\overline{Y_{i}}\right)\right)$ for each $i$. Suppose a mapping formula $\Psi$ is in the form $\psi(\bar{x}) \rightarrow \varphi(\bar{Y}, \bar{x})::$ annotation. Let $\mathcal{A}$ be a solution and let $\mathcal{I}=<\Delta^{\mathcal{I}},{ }^{\mathcal{I}}>$ be a model of $(\mathcal{T}, \mathcal{R}, \mathcal{A})$. Since $\mathcal{A}$ is a solution w.r.t. $\mathcal{X}$, then $\langle\mathcal{X}, \mathcal{A}\rangle$ satisfies all $\Psi_{\mathrm{s}}$ in $\Sigma_{\mathcal{S O}}$, i.e., for a tuple $\overline{s^{\prime}}$ in $\mathcal{X}$ such 
that $\mathcal{X} \models \psi\left(\overline{s^{\prime}}\right)$, there is a tuple $\bar{t}$ in Individual such that $\mathcal{A} \models \varphi\left(\bar{t}, \overline{s^{\prime}}\right)$ for each formula $\varphi(\bar{Y}, \bar{x})$. By the construction of $\mathcal{A}_{n}$, we know that if there is a tuple $\overline{s^{\prime}}$ in $\mathcal{X}$ such that $\mathcal{X} \models \psi\left(\bar{s}^{\prime}\right)$, then we add each assertion in the formula $\varphi\left(\bar{t}, \overline{s^{\prime}}\right)$ to $\mathcal{A}_{n}$ and $\mathcal{A}_{n}$ contains all and only the assertions which can be constructed from all mapping formulas $\varphi\left(\bar{t}, \bar{s}^{\prime}\right)$ and the axioms in TBox and RBox. Let $\mathcal{A}_{n}^{\prime}$ be the set of assertions instantiated from all formula $\varphi\left(\bar{t}, \overline{s^{\prime}}\right)$ and $\mathcal{A}_{n}^{\prime \prime}$ be the set of assertions added by applying propagation rules. Since $\mathcal{A} \models \varphi\left(\bar{t}, \overline{s^{\prime}}\right)$ for each formula $\varphi\left(\bar{t}, \overline{s^{\prime}}\right), \mathcal{A} \models \mathcal{A}_{n}^{\prime}$. Hence, the model of $\left.(\mathcal{T}, \mathcal{R}, \mathcal{A}), \mathcal{I}=<\Delta^{\mathcal{I}},{ }^{\mathcal{I}}\right\rangle$, is a model of $\left(\mathcal{T}, \mathcal{R}, \mathcal{A}_{n}^{\prime}\right)$. By the properties of the propagation rules, we know $<\mathcal{T}, \mathcal{R}$, $\mathcal{A}_{n}^{\prime}>\models \mathcal{A}_{n}^{\prime \prime}$; therefore, $\mathcal{I}$ is a model of $\left(\mathcal{T}, \mathcal{R}, \mathcal{A}_{n}\right)$. Since $\left(\mathcal{T}, \mathcal{R}, \mathcal{A}_{n}\right) \models p_{i}\left(h\left(\overline{Y_{i}}\right)\right)$ for each $i, \mathcal{I}=p_{i}\left(h\left(\overline{Y_{i}}\right)\right)$ for each $i$. By this we proved $(\mathcal{T}, \mathcal{R}, \mathcal{A})=p_{i}\left(h\left(\overline{Y_{i}}\right)\right)$ for each $i$. Therefore, $\bar{s} \in \underline{\operatorname{certain}}(Q, \mathcal{X})$.

\section{Conclusions}

We have studied the problem of translating an XML document into an instance of an OWL-DL ontology. However, we are aware that the problem of checking the satisfiability of the ontology and the consistency of the mapping formulas as well as answering conjunctive queries still has a very high complexity - NEXPTIME-complete for OWL-DL ontologies and EXPTIME-complete for OWL Lite ontologies [6,9]. We attempt to investigate some efficient algorithms for answering conjunctive queries over OWL-DL ontologies, probably incomplete but acceptable, in the future.

Acknowledgments: We are grateful to anonymous reviewers for offering valuable comments, corrections, and suggestions for improvement.

\section{References}

1. Y. An, A. Borgida, and J. Mylopoulos. Constructing Complex Semantic Mappings between XML Data and Ontologies. In ISWC'05, 2005.

2. M. Arenas and L. Libkin. XML Data Exchange: Consistency and Query Answering. In PODS'05, Baltimore, USA.

3. R. Fagin, P. G. Kolaitis, R. J. Miller, and L. Popa. Data Exchange: Semantic and Query Answering. In ICDT'03.

4. I. Horrocks and U. Sattler. Ontology Reasoning in the SHIQ(D) Description Logic. In IJCAI'01.

5. I. Horrocks and U. Sattler. A Tableaux Decision Procedure for SHOIQ. In IJCAI'05.

6. I. Horrocks and S. Tessaris. A Conjunctive Query Language for Description Logic Aboxes. In AAAI'00.

7. E. Prud'hommeaux, A. Seaborne. SPARQL Query Language for RDF. http://www.w3.org/TR/2004/WD-rdf-sparql-query-20041012/

8. R. Rodriguez-Gianolli and J. Mylopoulos. A Semantic Approach to XML-bases Data Integration. In ER'01.

9. S. Tobies. Complexity Results and Practical Algorithm for Logics in Knowledge Representation. PhD Thesis, LuFG Theoretical Computer Science, RWTHAachen, Germany, 2001. 